\title{
Genetically Similar Transduction Mechanisms for Touch and Hearing in Drosophila
}

\author{
Daniel F. Eberl, ${ }^{1}$ Robert W. Hardy, ${ }^{2}$ and Maurice J. Kernan ${ }^{1}$ \\ 1Department of Neurobiology and Behavior, The State University of New York at Stony Brook, Stony Brook, New York \\ 11794-5230, and 2Howard Hughes Medical Institute, University of California San Diego, La Jolla, California 92093-0649
}

\begin{abstract}
To test the effects of mechanosensory mutations on hearing in Drosophila, we have recorded sound-evoked potentials originating from ciliated sensory neurons in Johnston's organ, the chordotonal organ that is the sensory element of the fly's antennal ear. Electrodes inserted close to the antennal nerve were used to record extracellular compound potentials evoked by near-field sound stimuli. Sound-evoked potentials are absent in atonal mutant flies, which lack Johnston's organ. Mutations in many genes involved in mechanotransduction by tactile bristles also eliminate or reduce the Johnston's organ response, indicating that related transduction mechanisms operate in each type of mechanosensory organ. In addition, the sound-evoked response
\end{abstract}

is affected by two mutations that do not affect bristle mechanotransduction, beethoven (btv) and touch-insensitive-larvaB (tilB). btv shows defects in the ciliary dilation, an elaboration of the axoneme that is characteristic of chordotonal cilia. tilB, which also causes male sterility, shows structural defects in sperm flagellar axonemes. This suggests that in addition to the shared transduction mechanism, axonemal integrity and possibly ciliary motility are required for signal amplification or transduction by chordotonal sensory neurons.

Key words: Drosophila; mutant; hearing; touch; mechanoreceptor; mechanotransduction; chordotonal organ; cilium; axoneme
Many differently specialized sensory cells can transduce mechanical stimuli into changes in membrane potential, but it is not known whether their transduction mechanisms are related. A working classification of mechanosensors, which may have mechanistic implications, can be based on whether the transducing sensory cell is ciliated and whether the cilium is the site of transduction (Kernan, 1997). Examples of ciliary mechanoreceptors include some nematode amphid sensory neurons (Perkins et al., 1986; Kaplan and Horvitz, 1993) and the insect type I mechanosensory neurons that are the focus of this paper. Type I sensory organs form one of the two main classes of insect mechanosensory organs. They include one or more bipolar neurons, each bearing a ciliated sensory process, surrounded by specialized supporting cells. (Type II sensory cells are single, nonciliated multidendritic or bipolar neurons.)

Type I mechanosensory organs are further divided into two distinct subtypes (see Fig. 1): external sensory (es) organs and chordotonal (ch) organs (Keil, 1997). es organs have external cuticular structures, such as bristles, that can be deflected or deformed by touch, airflow, or proprioceptive stimulation. The outer segment of the neuronal sensory process, which contacts the external structure, is a ciliary derivative: it includes a short axoneme, but its distal part is occupied by a less regular array of microtubules, the tubular bundle. Effective stimulation is thought to occur when an extension of the cuticle compresses the ciliary membrane between an extracellular dendritic cap and the tubular bundle (Thurm, 1964). ch organs, in contrast to es organs, have no associated external structures. Instead, they lie under the cuticle and are stretched by flexion of the joint between two segments (Moulins, 1976; Shanbhag et al., 1992; Eberl, 1999). Their characteristic feature is the scolopale, a spindle-shaped cage enclosing an extracellular cavity, into which the ciliary outer segment extends.

Received April 6, 2000; revised June 5, 2000; accepted June 5, 2000.

This project was funded by a grant from the National Institute for Deafness and Communicative Disorders. We thank Charles Zuker and Norbert Perrimon for generous support during the initial phases of this work.

Correspondence should be addressed to Maurice Kernan, Department of Neurobiology and Behavior, The State University of New York at Stony Brook, Stony Brook, NY 11794-5230. E-mail: mkernan@notes.cc.sunysb.edu.

Dr. Eberl's current address: Department of Biological Sciences, University of Iowa, Iowa City, IA 52246-1324.

Copyright (C) 2000 Society for Neuroscience $0270-6474 / 00 / 205981-08 \$ 15.00 / 0$
Here, we investigate whether the same sensory transduction mechanism operates in both es and ch neurons. Previously, Drosophila mutations that eliminate mechanoreceptor potentials in external sensory bristles were identified in behavioral screens for touch insensitivity or severe uncoordination (Kernan et al., 1994). In an independent screen, mutants affecting sound-evoked courtship behavior have been isolated (Eberl et al., 1997). To determine whether either type of mutation affects transduction by the sensory neurons in Johnston's organ, the antennal chordotonal organ, we have recorded sound-evoked compound action potentials from the antennal nerves of the mutants and wild-type controls. Our results indicate that Johnston's organ is indeed an auditory receptor and provide evidence that related transduction mechanisms operate in external sensory and chordotonal neurons. Furthermore, we find that two mutations affecting only the chordotonal response show defects in the axonemal cytoskeleton, suggesting an additional role for ciliary action in mechanotransduction by these cells.

\section{MATERIALS AND METHODS}

Genetic strains and methods. Flies expressing the atonal (ato $\left.{ }^{1}\right)$ mutation were maintained as an ato ${ }^{1} / D f(3 R) p 13$ balanced stock; the mutation and deficiency stocks were generously provided by Andrew Jarman (University of Edinburgh, Edinburgh, Scotland). The EMS-induced unc, uncl, nompA, nomp $B$, nomp $C$, remp $A$, and tilB mutants have been described previously (Kernan et al., 1994); other nomp mutants were isolated in further iterations of the screen that produced the nompA-C mutants. (M. Kernan, D. M. Cowan, and R. Walker, unpublished data). unc and uncl mutants were maintained as $y w$ unc/FM4 X chromosome balanced stocks. All of the nomp and remp mutants were maintained as (nomp) cn bw/SM5 Cy $\mathrm{cn}^{2}$ balanced stocks. EMS-induced mutations on the second chromosome that affect auditory behavior have been described previously (Eberl et al., 1997). The 5P1 mutation from that study is here renamed beethoven $\left(b t v^{5 \dot{P} 1}\right)$. Other chromosomes, rearrangments, and mutations are described in FlyBase (1999). Flies were reared on standard media at $22-25^{\circ} \mathrm{C}$. unc and nomp mutant adults are severely uncoordinated and do not survive in culture vials; they were therefore collected from vials as pharate (uneclosed) adults and eclosed on filter paper dampened with a sucrose solution.

Electrophysiology. Auditory recordings from mutants and wild-type controls were performed $0-2$ d after eclosion; newly eclosed wild-type flies show a strong auditory response. For recording, flies, either unanesthetized or briefly chilled on ice, were introduced into $200 \mu$ l micropipette tips trimmed so that the front of the head and the antennae protruded. The head was stabilized with dental wax around the proboscis, and the micropipette tip was mounted on a movable stage. A pair of electrolytically sharpened tungsten electrodes were positioned in a double electrode 
holder so that one electrode could be inserted into the joint between the first and second antennal segments while the other was simultaneously inserted into the head capsule, both from the dorsofrontal aspect. The electrodes were connected to a DAM-50 differential amplifier (World Precision Instruments), and the unfiltered differential AC signal was amplified 1000-fold and sent to a MacAdios II/16 data acquisition board (GW Instruments) in a PowerMac 7500/80. The signal was sampled at a rate of $13.3 \mathrm{kHz}$ using Super Scope II software (GW Instruments). Power spectra of averaged responses were calculated with the Spectrum function of Superscope II: this function first weights the time series signal with a Hamming window, then calculates $\log 10$ of the spectral magnitudes of its fast Fourier transform (FFT)

Stimulus traces were generated with Super Scope II at 13,300 samples/ sec. Pulses were 2.5 cycles (i.e., $5 \mathrm{msec}$ ) of a $500 \mathrm{~Hz}$ sine wave, beginning with a phase of $90^{\circ}$, multiplied by an envelope of one cycle (trough to trough) of a sine wave of the same total length $(5 \mathrm{msec})$. Pulse song stimulus traces consisted of 5 pulses at $35 \mathrm{msec}$ intervals, with the first pulse initiating at $15 \mathrm{msec}$. The sine songs were $100 \mathrm{msec}$ tone bursts with linear on- and off-ramps. The computer-generated signals were amplified to a standard value with a Realistic MPA-30 amplifier (Radio Shack) and an 8 inch $4 \Omega$ Radio Shack speaker. The sound was delivered to the fly via 0.25 inch (inner diameter) Tygon tubing with one end mounted close to, but not touching, the speaker cone. A plastic pipette tip, cut to a $5 \mathrm{~mm}$ circular opening and plugged loosely with cotton to reduce echo, was inserted into the other end and mounted close to the fly; antennae were kept within the hemisphere circumscribed by the pipette opening to maintain near-field acoustic conditions (Bennet-Clark, 1971). A standard amplitude stimulus was used in all experiments unless noted otherwise, but the actual particle velocity and displacement at the antenna are unknown.

Electroretinograms were performed on flies mounted as for auditory recording. Saline-filled glass electrodes were inserted, just penetrating the surface of one eye and the dorsal head cuticle, and the differential voltage response was amplified 100-fold and recorded at 1000 samples/sec. An electrically driven shutter (Uniblitz) was used to deliver a $1 \mathrm{sec}$ white light stimulus from a DC-controlled halogen light source (Fostec) via an optic fiber bundle. A single, constant stimulus light intensity was used; recordings from each fly were made both in ambient room light and in near darkness. Recordings were made from at least three flies for each mutant genotype.

Courtship behavior. Behavioral audiograms were measured as described (Eberl et al., 1997). Briefly, genotypic groups of six males, whose wings had been removed earlier, were placed in the cells of the test chamber described previously. Computer-generated pulse song was presented incrementally, for $30 \mathrm{sec}$ at each intensity increment. Courtship behavior was quantified by scoring at $3 \mathrm{sec}$ intervals the number of males in a group that are involved in courtship. The 10 scores resulting from each intensity increment were summed to give the courtship index for that intensity. The maximum possible courtship index with six flies per group is 60 . btv homozygotes and heterozygous sib control flies were obtained directly from the $w ; b t v 40 A-G 13 / C y O$ stock. To approximate the same genetic background for tilB as for btv, we crossed $y w$ tilB/FM4 females to $w$; $40 A-G 13$ males and tested the $y w$ tilB male offspring. The control for tilB was the $y w$ background chromosome on which the tilB mutations were induced; this was also crossed to the $w ; 40 A-G 13$ stock for behavior testing.

Histology. Heads were dissected, by removing the proboscis and ventra tracheal sacs, in PBS $+0.1 \%$ Triton X-100 and transferred to $2 \%$ glutaraldehyde in PBS on ice. An equal volume of $4 \% \mathrm{OsO}_{4}$ in PBS was added to the heads, which were then incubated on ice for $30 \mathrm{~min}$. Heads were rinsed with cold PBS, then further fixed with $4 \% \mathrm{OsO}_{4}$ in PBS for 1-2 hr, followed by dehydration in an ethanol series (30, 50, 70, 90, 100\% ethanol; dilutions in PBS). The ethanol was replaced with propylene oxide (three 10 min washes at room temperature). Spurr's low-viscosity embedding resin (Polysciences, Warrington, PA) was added in equal volume and incubated overnight. This mixture was replaced with pure resin, and the heads were incubated for $4 \mathrm{hr}$. The heads were positioned in resin in silicone rubber molds, baked overnight at $75^{\circ} \mathrm{C}$, and sectioned at 1-8 $\mu \mathrm{m}$. Sections were photographed under oil using phase-contrast or Nomarski optics.

Electron microscopy. Adult testes of 0- to 2-d-old flies were fixed and processed according to the method of Colley et al. (1991) except for that of Figure $7 D$, which was fixed and processed according to the method of Tokuyasu et al. (1972). All tissues were embedded in Spurr's medium (Polysciences). For electron microscopy of Johnston's organ, adult heads were fixed and sectioned as described above for visible microscopy. Before viewing, ultrathin sections of all tissues were stained in alcoholic uranyl acetate and lead citrate.

\section{RESULTS}

\section{Anatomy and operation of Johnston's organ}

The Drosophila antenna is a near-field sound receptor: it functions best in a zone within one wavelength of a vibrating source, where there is bulk air particle displacement. These conditions apply in the fly courtship song, which is produced by a male fly vibrating one extended wing in close proximity to a female. Although the total energy dissipated by a fly's wing is small, the near-field amplitude
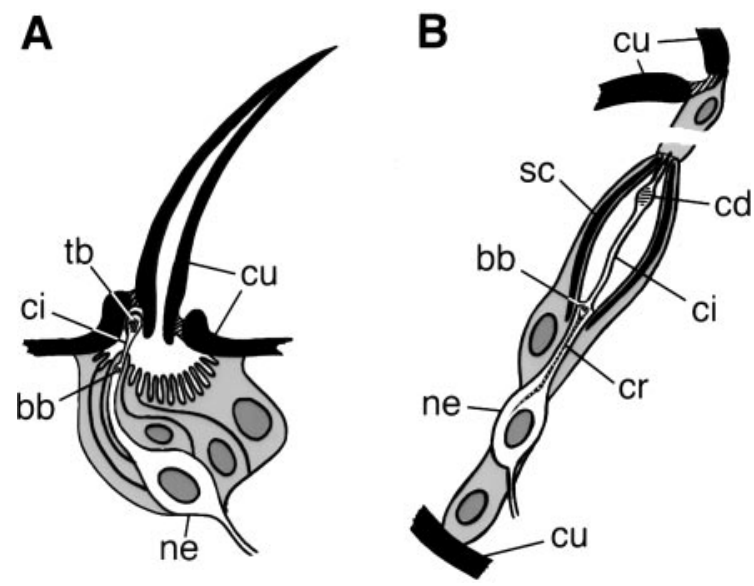

Figure 1. Schematic diagram of type I mechanosensory organs, drawn after Keil (1997) and Carlson et al. (1997). A, mechanosensory macrochaete bristle, an es organ. $B$, A single scolopidium of a chordotonal (ch) organ. An embryonic scolopidium is illustrated; scolopidia in the Johnston's organ have two sensory neurons and may differ in their attachment to the terminal supporting cells. Supporting cells (sheath, bristle, and socket cells in $A$; scolopale, cap, and ligament cells in $B$ ) and all nuclei are shaded; sensory neurons and extracellular spaces are white. Cuticular structures and the scolopale are solid black. $b b$, Basal body; $c d$, ciliary dilation; $c i$, cilium; $c r$, ciliary rootlet; $c u$, cuticle; ne, neuron; $s c$, scolopale; $t b$, tubular bundle.

when the male is close to the female's antenna can be $80-95 \mathrm{~dB}$ (Bennet-Clark, 1971). Air particle displacement moves the arista (the branched distal antennal segment (Fig. $2 A$ ) and the third antennal segment, relative to the second segment. These two segments are joined by a thin stalk, extending from the third segment, that fits inside a cup-shaped cavity in the second segment (Fig. 2B). The walls of the stalk and the cavity are reinforced by a honeycomb-like elaboration of the cuticle. Only the anteriomedial face of the stalk actually connects with the second segment; the antennal nerve exits the third segment through this connection.

Horizontal sections of the second segment (Fig. 2C,D) show Johnston's organ as an array of $\sim 100$ scolopidial elements that are attached basally to the outer wall of the second antennal segment and insert apically on the articulation connecting the second and third segments. Serial sections reveal the arrangement as a hollow conical bundle that occupies most of the second segment. All of the scolopidia in Johnston's organ appear to insert tangentially on the articulating membrane so as to be stretched, and thus stimulated, when the arista is rotated posteriorly.

In adult flies mutant for the proneural gene atonal, chordotonal organs, photoreceptors, and a subclass of olfactory receptors fail to develop because their precursors are not specified (Jarman et al., 1993, 1995; Gupta and Rodrigues, 1997). A sectioned second antennal segment from an atonal fly (Fig. $2 E$ ) shows it to be devoid of scolopidia, although the antennal nerve, composed of sensory projections from the more distal segments, is present.

\section{Recording sound-evoked potentials}

To examine the electrophysiological response of Johnston's organ, we recorded sound-evoked compound potentials from the antennal nerves of restrained intact flies. Extracellular potentials were recorded from an electrode inserted between the first and second antennal segments (Fig. $3 A$ ). At this point, the antennal nerve includes axons projecting from the chordotonal organs and mechanosensory bristles on the second antennal segment as well as projections from olfactory and other sensory organs in the more distal segments.

Sound stimuli were designed to mimic the sine wave and pulse phases of the fly's courtship song. In response to sine waves of 160 $\mathrm{Hz}$, the normal song frequency, a sustained response was seen, with multiple peaks corresponding to each stimulus cycle (Fig. $3 B$ ). At $500 \mathrm{~Hz}$ and higher frequencies, the peak amplitudes during sustained stimuli were reduced and poorly resolved at frequencies of 

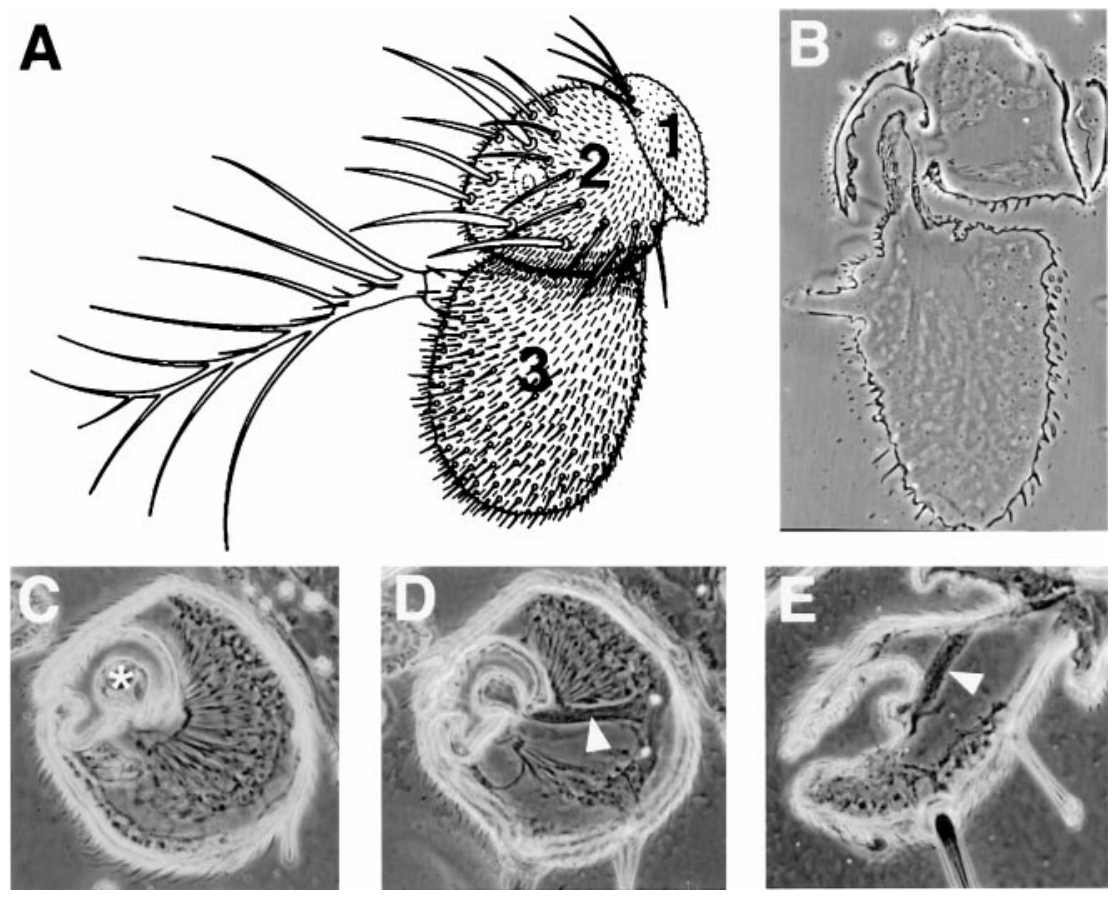

Figure 2. A, Frontal view of a right-hand Drosophila antenna [drawing by $\mathrm{P}$. Bryant reproduced from FlyBase (1999)]. The first, second, and third antennal segments are numbered. $B-E$, Phase-contrast images of sectioned antennae. $B$, Frontal section through the second and third antennal segments, in the same orientation as $A$. $C, D$, Two horizontal sections through the second segment, showing the array of scolopidia inserted on the articulation between the second and third segments. An asterisk in $C$ marks the stalk connecting the third segment to the second; the arrowhead in $D$ indicates the antennal nerve. $E$, Horizontal section through the second segment of an atonal mutant (ato $\left.{ }^{1} / D f(3 R) p 13\right)$. No scolopidia are present, but external mechanosensory bristles on the second segment are retained, as is the antennal nerve (arrowhead) comprising projections from the distal segments.
$750 \mathrm{~Hz}$ or greater (data not shown). Spectral analyses of the averaged responses to sine stimuli (Fig. $3 E$ ) show peaks at multiples of each stimulus frequency, reflecting a complex response evoked by each stimulus cycle. Single traces recorded from wild-type flies in response to a train of five pulses show clear fluctuations in extracellular potential after each pulse (Fig. 3C). The latency of the observed response (Fig. 3D), mainly attributable to the time taken for the stimulus to travel through a sound tube, excludes the possibility of an electrical artifact. Varying tube lengths showed a linear relationship with latency, and extrapolating to zero travel time indicated a maximum residual latency of $1.2 \mathrm{msec}$. Averaging of multiple consecutive traces showed conservation of the overall waveform between individual responses, although individual peak amplitudes varied. Wild-type female and male flies and Y chromosome-deficient (XO) males all gave similar responses to pulse and sine stimuli.

To identify the source of these signals, we recorded the soundevoked response from atonal mutant flies, which lack chordotonal organs (Fig. 2E). No sound-evoked potential was seen in atonal flies in response to any stimulus, even when increased stimulus intensities were used. Thus, Johnston's organ is the probable source of the sound-evoked potentials in wild-type flies. atonal mutants also lack other sensory cells, including photoreceptors (Jarman et al., 1994) and a subset of antennal olfactory bristles (Gupta and Rodrigues, 1997); the results do not exclude the possibility of an indirect effect on the sound-evoked response via these or other cells.

\section{Mutations affecting transduction in both es and ch organs}

Behavioral screens for mechanosensory defects have identified a set of mutations that affect mechanotransduction in es organs (Kernan et al., 1994). These include recessive mutations in the uncoordinated (unc) and uncoordinated-like (uncl) genes on the $\mathrm{X}$ chromosome and in several no mechanoreceptor potential (nomp) or reduced mechanoreceptor potential (remp) genes on the second chromosome. All of these mutations cause a distinctive type of uncoordination, in which legs are frequently crossed and wings are held up or out. In the most severe cases, mutant adult flies are incapable of walking or righting themselves; however, they still show vigorous, albeit uncoordinated, activity spontaneously or in response to light or harsh mechanical stimuli, indicating that general neuromuscular excitability is retained. Mechanosensory receptor poten- tials can be recorded directly from single bristle neurons as changes in transepithelial potential evoked by mechanical stimuli (Wolbarsht and Dethier, 1958; Wolbarsht, 1960). In unc, uncl, and nomp mutants, mechanoreceptor potentials are reduced or absent in thoracic macrochaete bristles (Kernan et al., 1994). The global touch insensitivity and severe uncoordination characteristic of this class of mutation suggest that smaller tactile and proprioceptive hair-plate bristles are also affected.

To determine whether these mutations also affect transduction by the antennal chordotonal organ, we attempted to record soundevoked potentials from the antennal nerves of representative mutant genotypes, using a standard pulse stimulus. We tested four mutants-unc, uncl, nomp $A$, and nompB -in which bristle mechanoreceptor potentials are invariably absent, and seven mutantsnотр $C$, потрE, nотрF, nотрI, nотрJ, remp $A$, and rempD-in which the bristle mechanoreceptor potential amplitudes are usually absent or are reduced. The results were striking: all four genotypes that eliminate bristle receptor potentials also eliminated the soundevoked response (Fig. 4). Among the mutations that reduce or variably eliminate bristle receptor potentials, nompF and rempA showed no sound-evoked response, whereas nompE, nompI, and nompJ reduced the response to near-background levels. nompC and rempD showed less severe reductions in response amplitude. Five of the mutants (nompE, nompF, nompI, nompJ, and rempD) are each represented by homozygotes for a single allele; for these genotypes, the possibility of different phenotypes being caused by multiple linked mutations cannot be excluded.

These mutants are all severely uncoordinated and consequently do not survive long as adult flies; all recordings were performed within 1-2 d of eclosion. To test whether a general debilitation affected the sound-evoked response, we also tested a mutagenized line, 5-68, that shows a similar degree of uncoordination but that has normal bristle electrophysiology (data not shown). The soundevoked potential amplitudes from this line were in the normal range (Fig. 4). To check for more general defects in sensory cell excitability, we also recorded electroretinograms (ERGs) from flies mutant for unc, uncl, and each of the nomp and remp genes. The ERG records a sustained electrical response of retinal photoreceptors to a light stimulus; transient components at the stimulus onset and offset reflect synaptic activity in the optic lamina (Pak et al., 1969). All mutants tested except rempD showed electroretinogram responses indistinguishable from wild-type controls (data not 

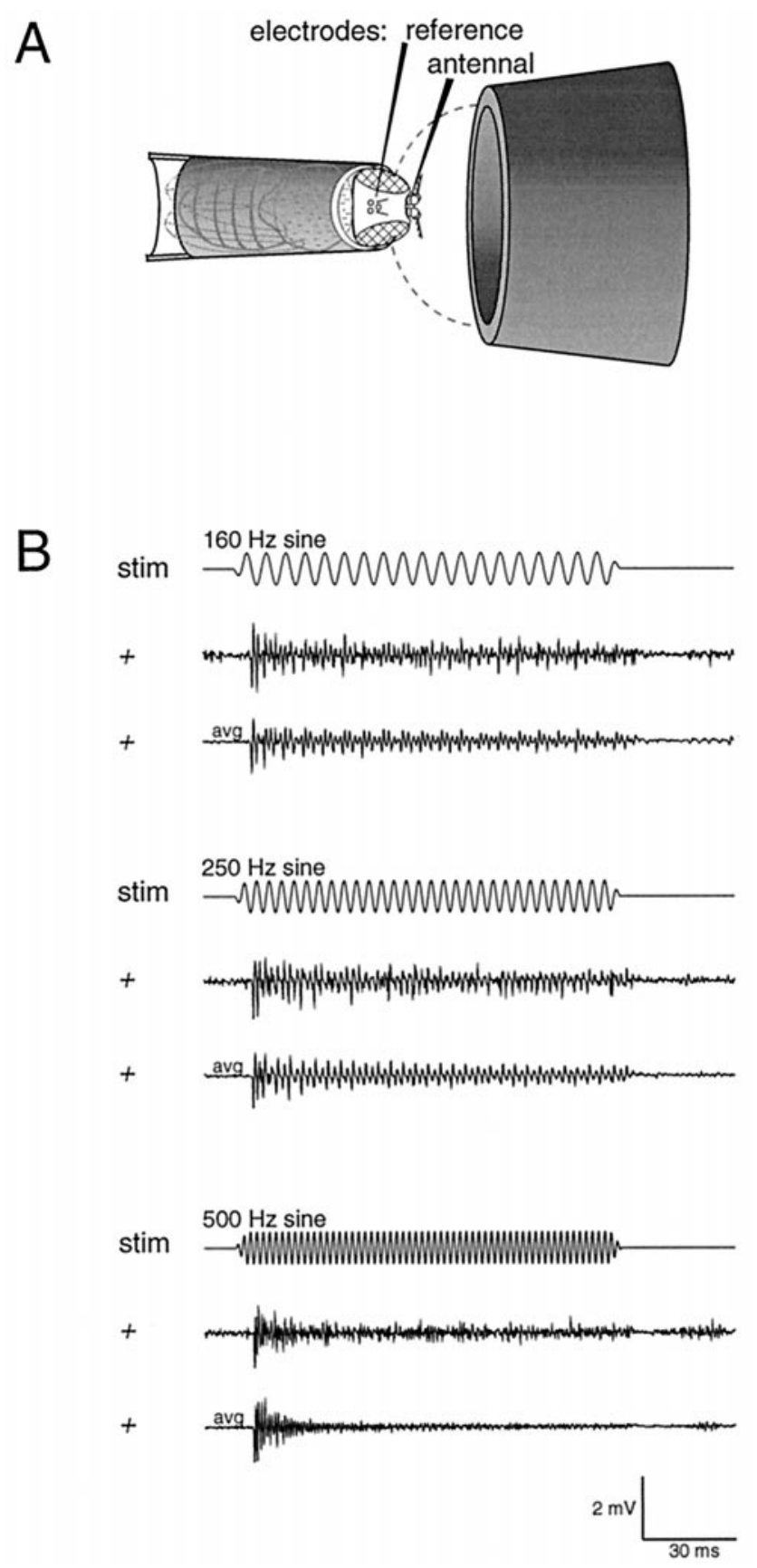
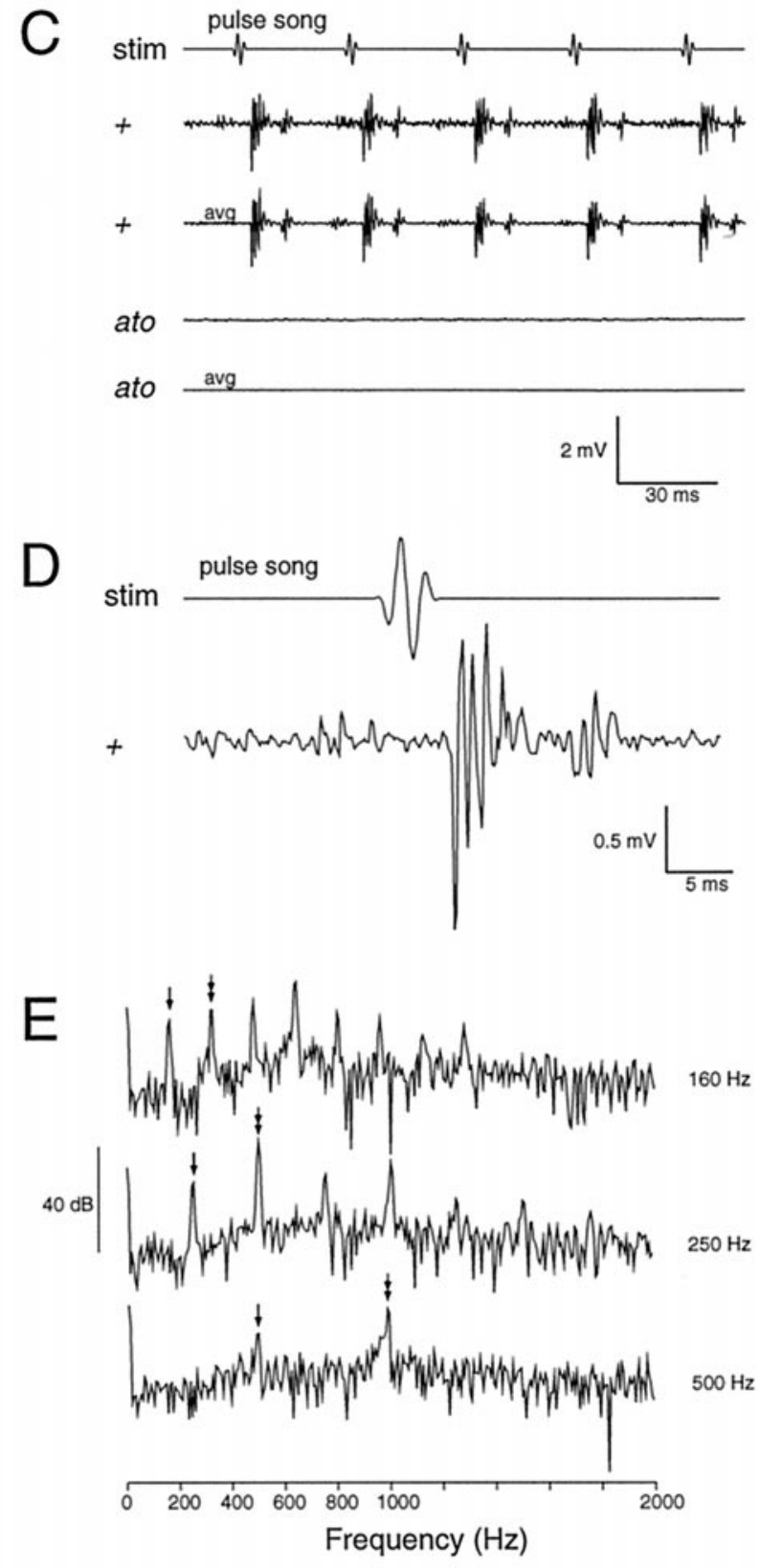

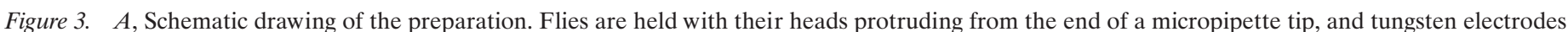

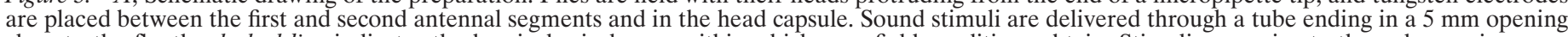

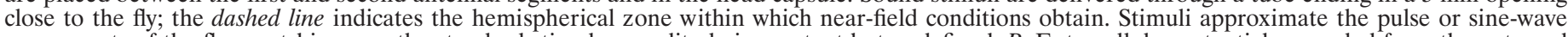

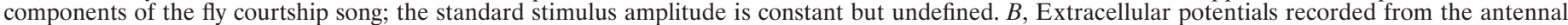

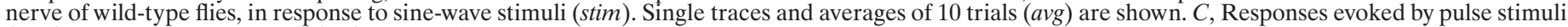

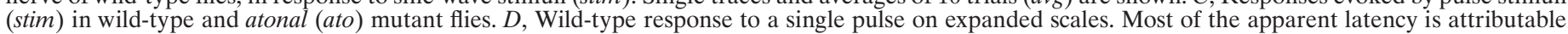

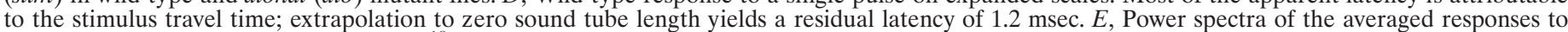

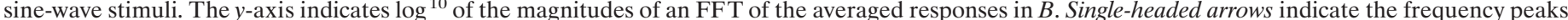

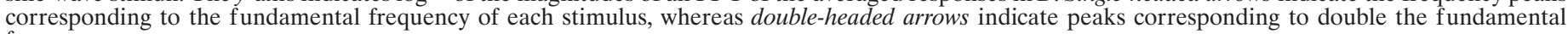
frequency.

shown), indicating that phototransduction and associated synaptic activity are normal in these mutants. rempD mutants had reduced ERG amplitudes and off-transients: in this one case, a more general defect may affect several sensory modes.

\section{Mutations affecting transduction in ch but not es organs: btv and tilB}

The preceding results imply that bristles and chordotonal organs are functionally similar, but they do not preclude properties specific to either type of sensory organ. Mutations specifically affecting chordotonal organs would be expected to have an auditory defect. A set of candidate auditory mutants was isolated by screening for lines in which amplified courtship song failed to elicit male courtship behavior (Eberl et al., 1997). Thirteen independently isolated homozygous courtship-defective lines were tested for soundevoked antennal nerve potentials. One line, $5 \mathrm{P} 1$, which shows a reduction in sound-evoked courtship behavior (Fig. $5 A$ ), gave no response to the standard pulse stimulus. Increasing the stimulus 


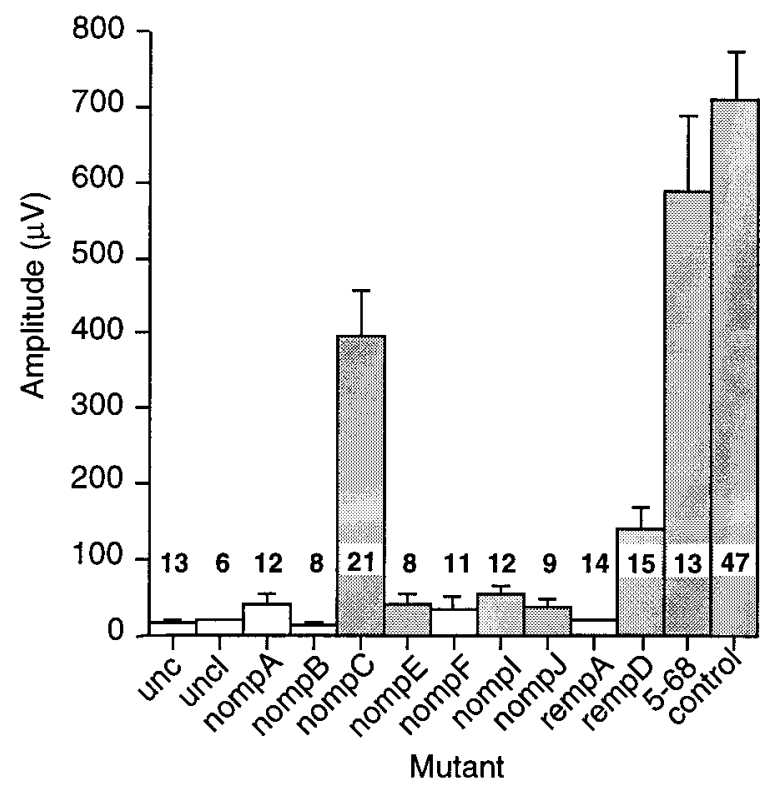

Figure 4. Sound-evoked potential amplitudes in wild-type and mutant flies. Data are the maximum amplitudes of the averaged response of a single antenna to 10 trials of a standard five-pulse stimulus. Shaded bars indicate those genotypes in which a response to individual pulses was distinguishable in some or all records; the values in other genotypes reflect baseline noise. Error bars are SEMs. The number of antennae recorded from each genotype is indicated. Mutant genotypes: unc: $u n c^{25} / Y$, unc ${ }^{27} / Y$, and $D f(1) S 54 /$ $D f(1) B 57$ (data pooled); uncl: $D f(1) G A 33 / D f(1) E A 113$; nompA: nomp $A^{1} \mathrm{cn}$ bw/nomp $A^{2} \mathrm{cn}$ bw; nompB: nompB ${ }^{1} \mathrm{cn}$ bw/nompB ${ }^{2} \mathrm{cn}$ bw and nompB ${ }^{1} \mathrm{cn}$ $b w / n o m p B^{3} c n$ bw (data pooled); nompC: nomp $C^{1} c n b w / D f(2 L) c l^{\mathrm{h} 2}$; nompE: nomp ${ }^{1}$ cn $b w$ homozygotes; nompF: nomp $F^{1}$ cn bw homozygotes; nompI: nompI ${ }^{1}$ cn bw homozygotes; nompJ: nompJ ${ }^{1}$ cn bw homozygotes; rempA: remp $A^{1}$ cn bw/remp $A^{2}$ cn bw; rempD: rempD ${ }^{1}$ cn bw homozygotes; 5-68: 5-68 $c n$ bw homozygotes. Controls are pooled heterozygotes for nomp or remp mutations and the balancer second chromosome $\mathrm{CyO}$; they are sibs of the mutant nomp or remp homozygotes.

intensity yielded a tiny response (Fig. 5B). Two chromosomal deletions, Df(2L)TW201 and Df(2L)TW119, which overlap at the cytogenetic interval 36E1-3, both fail to complement this electrophysiological phenotype and thus place the affected locus, which we have named beethoven (btv), in this interval. Flies carrying both deletions in combination are viable and also show a very reduced but not completely absent sound-evoked potential, similar to that seen in $b t v^{5 \mathrm{P} 1}$ homozygotes (data not shown).

The other 12 courtship-defective lines showed no apparent defect in sound-evoked potentials. Six lines had a more severe behavioral phenotype than $b t v$ mutants (Eberl et al., 1997), implying that the failure to evoke courtship behavior is caused by defects other than auditory transduction rather than a hypomorphic or less penetrant transduction defect. Because the courtship screen required adult flies to be motile and relatively coordinated, it is unlikely that flies with the comprehensive mechanosensory defects characteristic of the nomp and unc mutants would have been recovered in this screen. Indeed, btv mutants showed normal mechanoreceptor potentials in thoracic macrochaete bristles (data not shown).

A second gene affecting chordotonal organs, touch-insensitivelarva $B(t i l B)$, is identified by two alleles that were isolated in a screen for reduced larval response to touch and were cytogenetically mapped to position 20A4. Unlike the unc and uncl mutants, which were isolated in the same screen, tilB mutant adults show only a slight motor uncoordination and have normal bristle receptor potentials (Kernan et al., 1994). tilB ${ }^{1 / Y}$ and $t_{i l B}{ }^{2} / Y$ hemizygous males show a complete absence of sound-evoked courtship behavior (Fig. 5A). No sound-evoked antennal nerve potentials were observed in tilB/Y males (Fig. $5 B$ ) or in tilB $^{1} /$ tilB $^{2}$ heterozygous females at any stimulus intensity.

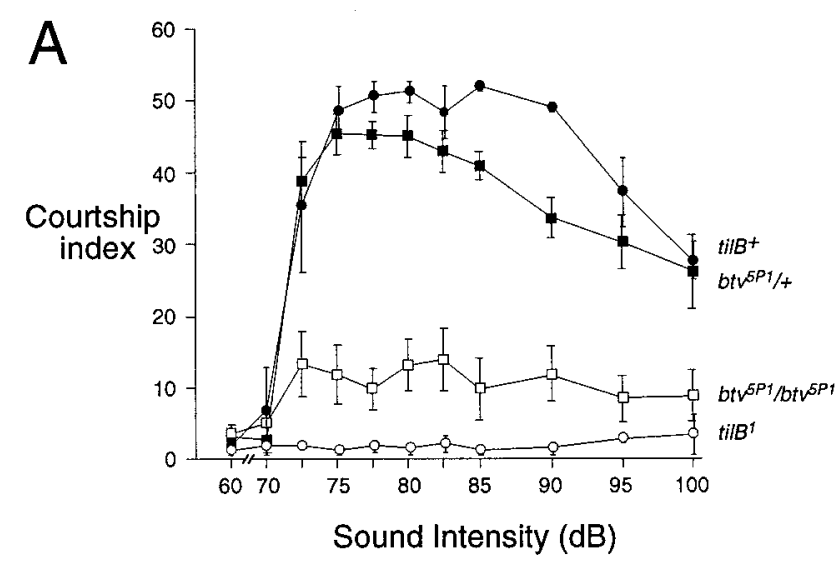

B
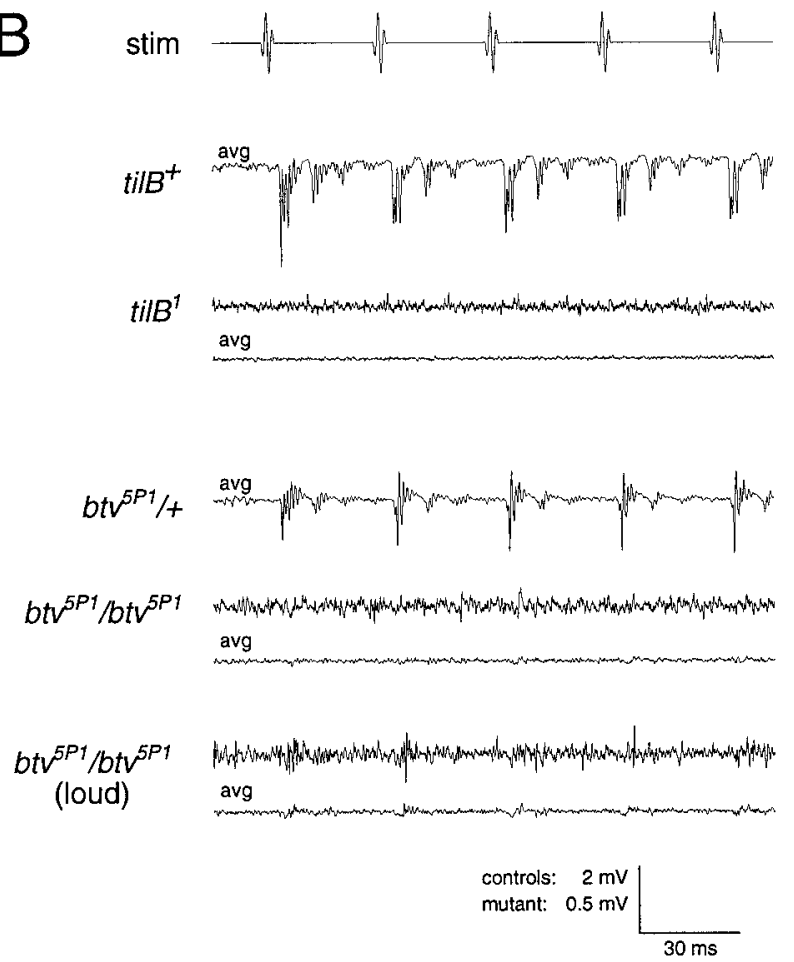

Figure 5. Auditory defects in the tilB and btv mutants. $A$, Behavioral response to courtship pulse song in $t i l B$ and $b t v$ mutants and wild-type controls. Groups of six male flies of each genotype were exposed to a series of $30 \mathrm{sec}$ intensity increments of pulse song. The number of flies performing courtship behavior was recorded 10 times during each $30 \mathrm{sec}$ increment and summed to give the courtship index. Filled symbols, Wild-type controls; open symbols, mutants. B, Sound-evoked potentials from tilB and btv mutants and wild-type controls. Single and averaged responses were recorded as in Figure 3. Only the averaged responses are shown for the wild-type controls; these are shown on a reduced scale. An increased stimulus amplitude was used to evoke the final pair of btv records.

\section{Axonemal defects in btv and tilB}

Examination of sectioned btv and tilB mutant antennae by light microscopy showed no gross defects in Johnston's organ. To search for ultrastructural defects, ultrathin sections of Johnston's organ were examined by electron microscopy (Fig. 6). Prominent in sections of a wild-type scolopidium are the scolopale and the cilia and ciliary rootlets of the two sensory neurons (Fig. 6A). The cilium of a ch neuron differs from that of an es neuron in having an axonemal cytoskeleton throughout its length. At approximately three-fourths of their length toward their distal tips, the cilia are dilated, enclosing paracrystalline inclusions (Fig. 6 $A, B$ ) [see also Uga and Kuwabara (1965) and Hallberg (1984)]. In the btv deletion 

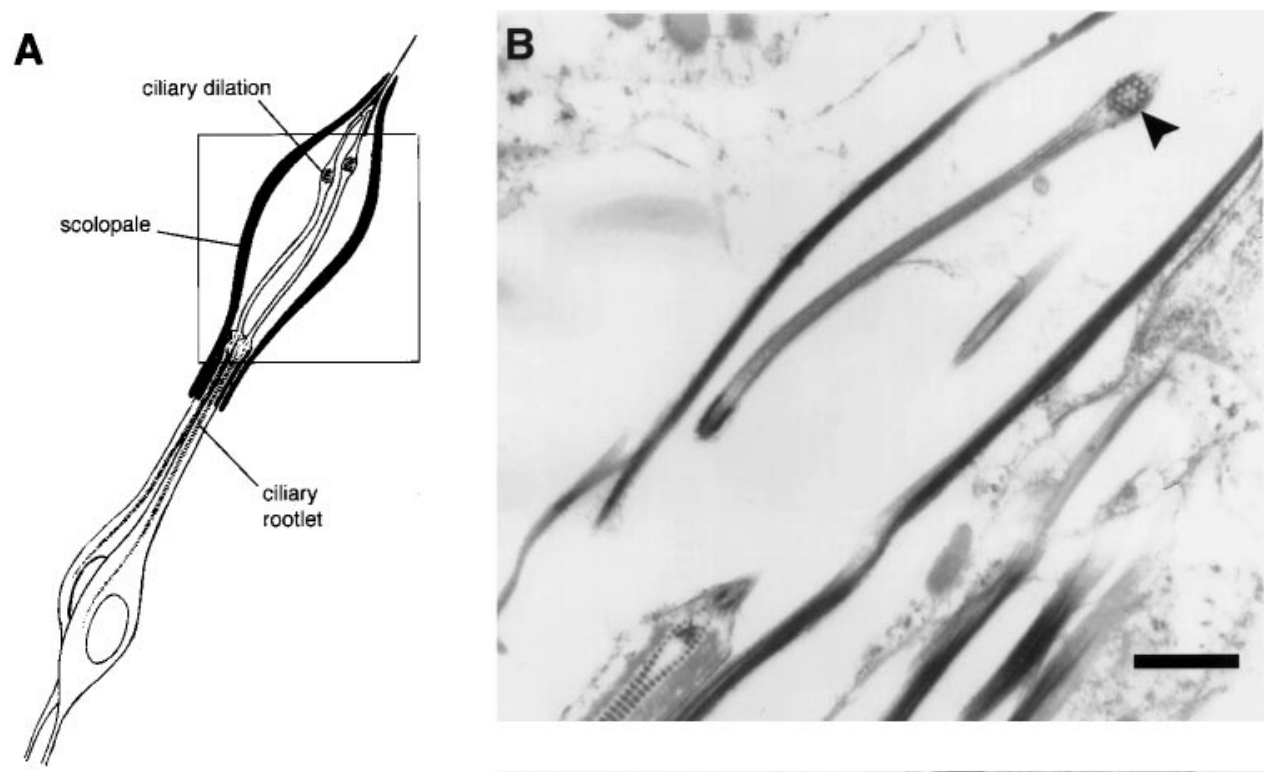
Figure 6. Ciliary ultrastructure in the
Johnston's organ of wild-type and $b t v$ mutant flies. $A$, Sketch of a single antennal scolopidium showing the pair of sensory neurons and the scolopale in longitudinal section; the scolopale cell and other supporting cells are omitted. The approximate area shown in Figure $5, B$ and $D$, is outlined. $B$, Longitudinal section through a wild-type (Canton-S) scolopidium. Most of one cilium including the ciliary dilation (arrowhead) is included in this section; the basal body attachment and ciliary rootlet of the other neuron are also visible. Scale bar, $1 \mu \mathrm{m}$. $C$, Pair of ciliary dilations in a wild-type scolopidium. Scale bar, $200 \mathrm{~nm}$. $D$, Longitudinal section through two btv mutant scolopidia [heterozygous for the overlapping deletions $D f(2 L) T W 119$ and $D f(2 L) T W 201]$. The regular substructure of the ciliary dilations (arrowheads) is absent. Scale bar, $1 \mu \mathrm{m}$.
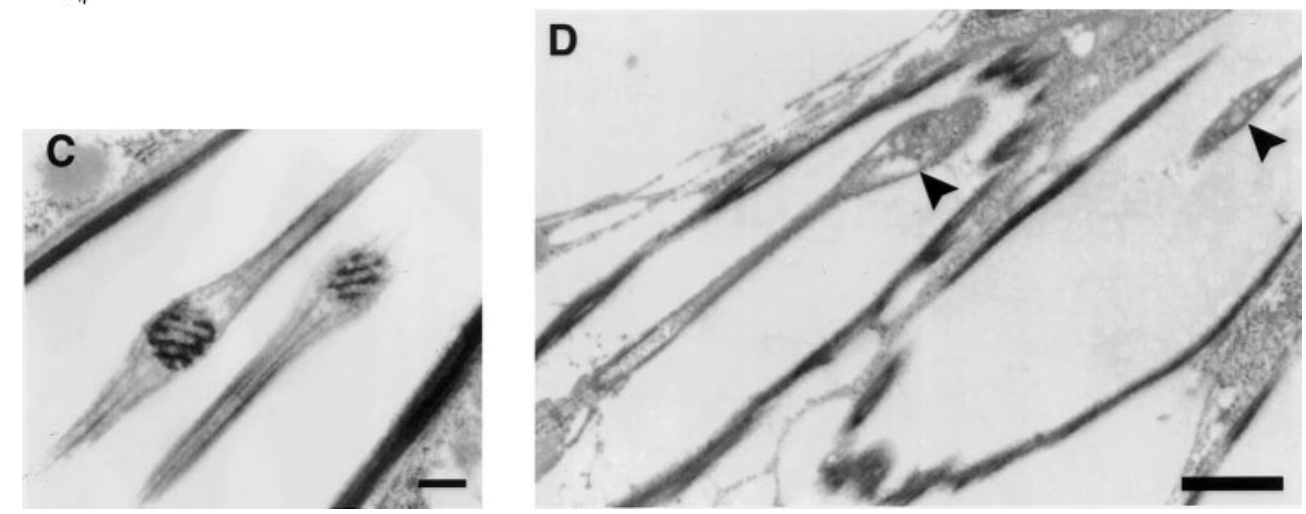

genotype, the ciliary dilations are enlarged, and the paracrystalline inclusions are absent and replaced by vacuoles (Fig. 6C).

No ultrastructural defect was observed in Johnston's organ of tilB mutants. Nevertheless, a possible axonemal defect was suggested by another phenotype of both $t i l B$ alleles-male sterility. Comparison of the reproductive organs from mutant and control males showed that in tilB the testes are present and normal in appearance, but although $t i l B$ sperm elongate and reach a late stage of differentiation, they are not transferred to the seminal vesicle and never become motile.

When differentiating spermatids in tilB and wild-type males were examined by electron microscopy, a defect was indeed found in flagellar axonemes. Wild-type Drosophila spermatid axonemes (Fig. $7 A, C$ ) have a central pair of microtubules surrounded by nine sets of outer microtubule doublets and accessory microtubules. In wild-type axonemes, each microtubule doublet bears inner and outer dynein arms projecting from the A subtubule as well as a nexin link between each pair of AB doublets. The dynein arms are the dynamic elements of sperm motility, whereas the nexin links bind the ring of doublets together. In $t i l B$ mutants, both dynein arms are missing, and the nexin link may be as well. In tilB spermatids (Fig. $7 B, D$ ), some axonemal profiles are split; this phenotype varies from a single break in the ring of microtubule doublets to a complete separation of some of the doublets, together with their accessory tubules and associated radial spokes. Split axonemes are observed in elongation stage spermatids, a stage preceding the addition of dynein arms to wild-type axonemes. Thus both absence of dynein arms and split axonemes may result from the absence of another component of the axoneme or a specific defect in its assembly.

In contrast to $t i l B$, males homozygous for $b t v^{5 \mathrm{P} 1}$ have motile sperm and are fertile, as are $b t v^{5 \mathrm{P} 1} /$ deletion heterozygotes. How- ever, males with a deletion overlap for the btv region are sterile, with nonmotile sperm and occasional axonemal breaks similar to those seen in $t i l B$ mutants (data not shown). The dynein arms, however, are retained in this genotype. Male sterility may be the consequence of a btv null genotype, but because the deleted interval may include a separate male fertility locus, this phenotype cannot yet be unambiguously ascribed to btv.

\section{DISCUSSION}

We draw three main conclusions from these data. First, Johnston's organ is the Drosophila auditory receptor. Second, a mechanism requiring the $u n c, u n c l$, and many nomp gene products transduces mechanical stimuli in both external sensory and chordotonal organs. Third, the btv and tilB mutations reveal a more stringent requirement for axonemal integrity and possibly ciliary motility in chordotonal organs.

\section{Johnston's organ is the Drosophila auditory organ}

Several types of evidence suggest that the second antennal segment can function as an auditory organ. First, the arista and third antennal segment rotate easily relative to the second segment and vibrate visibly during acoustic stimulation (data not shown); the Johnston's organ scolopidia are well placed to detect this movement. Second, immobilizing or removing antennal segments affects sound-evoked behavior patterns (Manning, 1967a,b). Third, soundevoked potentials have been recorded from Johnston's organ in mosquito (Tischner, 1953) and from the antennal nerve in the blowfly Calliphora erythrocephala (Burkhardt and Gewecke, 1965) and in Drosophila funebris (Ewing, 1978). Here, we find that atonal mutants, which lack chordotonal organs but retain most other antennal sensory organs, show no sound-evoked potentials. Although an indirect effect of ato on the auditory response cannot be 

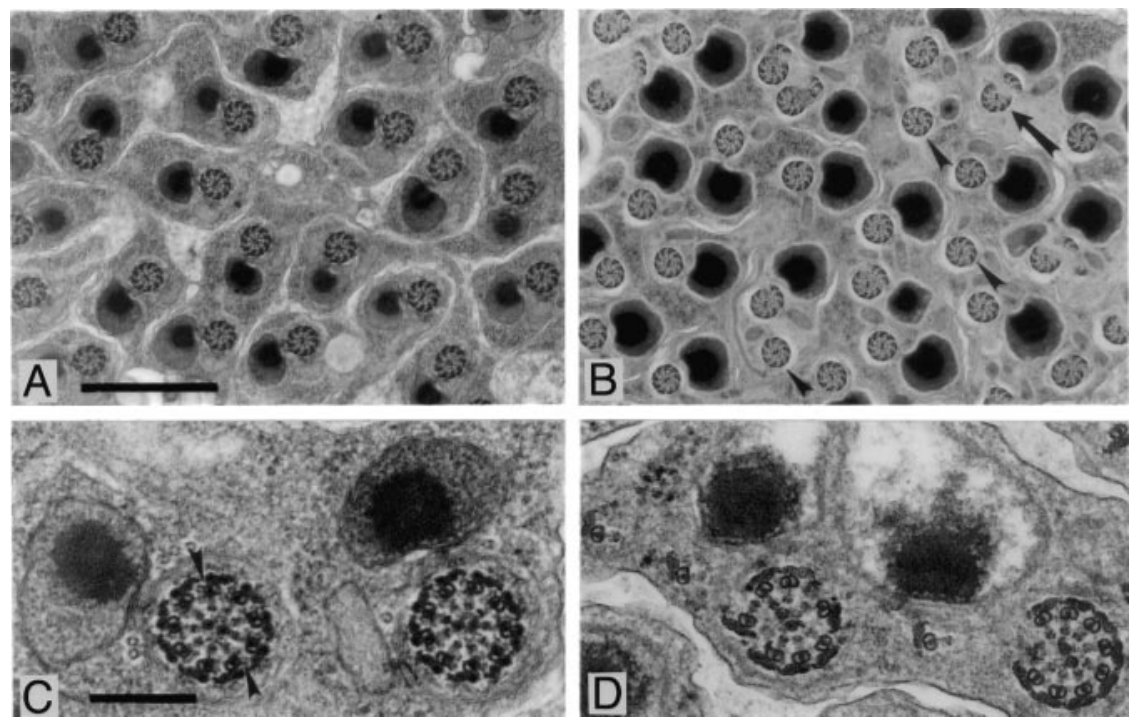

Figure 7. Transverse sections of testes showing postelongation spermatid flagellar profiles. $A$, Wild-type control $(y w / Y)$. Spermatids elongate in syncitial bundles of 64 spermatids; part of one bundle is shown here. The dark area associated with each axoneme is a condensing mitochondrial derivative. Scale bar, $1 \mu \mathrm{m}$. $B$, tilB $\left(y w\right.$ tilB $\left.^{1} / Y\right)$. Arrow indicates a split axoneme; 31 of the 64 spermatids in the complete bundle had split axonemes. The arrowheads indicate axonemes with abnormal or missing mitochondrial derivatives. $C$, Wild type. Arrowheads indicate the outer dynein arms extending between the A subtubule and the satellite tubules. Scale bar, $200 \mathrm{~nm}$. $D$, tilB , showing fragmented axonemes without inner and outer dynein arms. excluded, the most likely interpretation is that Johnston's organ is the source of the sound-evoked potentials in wild-type flies.

The Drosophila Johnston's organ contains $\sim 100$ scolopidial units; the observed sound-evoked potentials are likely the aggregate product of many or all of these units. The presence of multiples of the stimulus frequency in the response to a sine wave suggests that different units may be responding to different phases of the stimulus. However, there is no obvious anatomical basis for this complexity: all of the scolopidia are arranged so that they will be stretched together as the antenna rotates. Alternatively, each of the two neurons in a single scolopidium may be sensitive to different phases of the stimulus. Antennae responded sensitively and with a consistent latency to single pulses or to the onsets of longer stimuli. These properties may reflect the need to preserve the interpulse interval (IPI) information contained in the pulse mode of courtship song. Pulse song is the more effective mode for eliciting courtship behavior in male groups (von Schilcher, 1976; Eberl et al., 1997), and the IPI is the parameter that distinguishes species-specific courtship songs (Bennet-Clark and Ewing, 1969; Kyriacou and Hall, 1986).

\section{A related mechanism for mechanotransduction in type I mechanosensory organs}

Because unc and nomp mutations affect mechanically evoked receptor potentials in external sensory bristles without causing gross defects in sensillar development, they identify genes required for the construction or operation of mechanosensory transducers (Kernan et al., 1994). The concordant effect of mutations in most of these genes on the Johnston's organ response implies that chordotonal organs use many of the same components. Interestingly, mutations in nompC, which encodes an ion channel that is intimately involved in bristle mechanotransduction (Walker et al., 2000), are a partial exception, because their effect on the soundevoked response is less dramatic than on bristle receptor potentials and currents (Kernan et al., 1994; Walker et al., 2000). The NompC channel may function only in a subset of the Johnston's organ scolopidia, or it may contribute a smaller proportion of the mechanotransducer current in chordotonal neurons than in bristle neurons.

The auditory courtship mutants provide an informative contrast to the tactile/uncoordinated mutants; 12 of 13 mutants had no effect on the sound-evoked electrophysiological response. The courtship screen required viable, coordinated adult flies and probably excluded general mechanotransduction mutants. Recovery of only one auditory transduction mutant in this screen is thus consistent with a largely similar mechanism in chordotonal organs and proprioceptive bristles. The 12 courtship mutants with normal audi- tory transduction may instead be defective in downstream auditory processing or in courtship behavior.

Drosophila es and ch organs are morphologically quite different. The stimulus that is thought to depolarize some es neuronsorthogonal compression of the ciliary membrane against the tubular bundle — seems quite different from the longitudinal stretch that probably stimulates ch organs, but the precise forces operating at the molecular scale on the transducing components in either sensillum cannot yet be specified, particularly because the transducer location in ch organs is unknown. Identifying and locating the nomp gene products in each type of sense organ will help to resolve the genetic similarities and mechanical differences in their operation.

A single type I transduction mechanism in type I mechanosensors is consistent with the likely evolutionary origins of the es and ch subtypes. Ciliated sensory organs are found throughout the Arthropoda, but chordotonal organs with scolopidia are restricted to insects and crustaceans (Moulins, 1976; Merritt, 1997). Although there is a marked distinction between the es and ch subtypes in dipteran insects, a more diverse spectrum of ch types is found in other insect orders and in crustaceans, including forms that have both a scolopale and a distal ciliary segment similar to the tubular bundle of es organs. It is not clear which of these structural features is primitive (Keil, 1997), but it is likely that an ancestral type I sensillum had features intermediate between the es and ch types (Merritt, 1997).

Drosophila and other insects also bear type II, nonciliated peripheral neurons (Bodmer and Jan, 1987). Some may be mechanosensory, but their electrophysiological properties have not been defined; we do not know whether they are affected by nomp and unc mutations. An ion channel subunit expressed by some type II cells (Adams et al., 1998; Darboux et al., 1998) is related to the channel that functions in the nonciliated "body touch" cells of the nematode Caenorhabditis (Driscoll and Chalfie, 1991; Huang et al., 1995; Tavernarakis and Driscoll, 1997). Ciliated mechanosensitive cells in Caenorhabditis are not affected by mutations in this channel ( $\mathrm{J}$. Kaplan, personal communication), implying that a different transduction mechanism operates in nematode ciliated neurons. The expression of a Caenorhabditis NompC homolog in a subset of sensory cilia (Walker et al., 2000) suggests that this mechanism is conserved across phyla.

\section{Ciliary action in chordotonal organs: a role in transduction?}

The limited sensory effects of the btv and tilB mutations imply that they affect an activity required for transduction by ch organs but not by es organs. We believe dynamic ciliary action to be this extra 
function. Chordotonal cilia have an extended axoneme and are free to move within the lumen of the scolopale, whereas the ciliary segment of es neurons has only an abbreviated axoneme and is closely surrounded by a dendritic cap. Thus, active ciliary movement may be possible only in ch organs. Ciliary bending has previously been reported in stimulated grasshopper femoral chordotonal organs (Moran et al., 1977).

Mutations in both tilB and btv cause axonemal defects and are likely to interfere with ciliary action. In tilB mutants, both sperm motility and chordotonal organ transduction are eliminated. The absence of dynein arms in tilB sperm axonemes is sufficient to explain their immotility. However, chordotonal cilia are not functionally identical to sperm flagella (for instance, females and XO males lack Y-encoded sperm axonemal components but are not deaf), so we cannot be sure that tilB also causes ciliary immotility in Johnston's organ. The fact that an axonemal defect silences the neuron without causing a gross structural defect in the chordotonal cilia implies that these cilia do more than passively transmit the mechanical stimulus. In $b t v^{5 \mathrm{P} 1}$ mutants, the ciliary dilation, a structure unique to chordotonal organs, is defective. Sperm axonemal motility is retained in this mutant (although perhaps not in btv null males). Thus, scolopidial ciliary motility may require an intact ciliary dilation, or this structure may have a more specific role in the transduction mechanism.

What role could ciliary action play in sensory transduction? The probable effective stimulus for a scolopidium is stretch along its long axis, the direction in which the cilium extends, and the stimulus is delivered via a cap attached to the tip of the cilium. Active bending or writhing of the cilium will shorten its overall length and increase the tension in all elements to which it is attached. Thus, it could act to amplify the stimulus or to maintain an optimal tension on the transduction mechanism.

This postulated role of the cilium is functionally analogous to the activity of mammalian outer hair cells, which boost the cochlear response by changing shape in response to depolarization (for review, see Dallos, 1992). As a consequence of this or other mechanical activities, vertebrate cochleae generate spontaneous or evoked otoacoustic emissions. Such otoacoustic emissions have also been recorded from moth and locust tympanal ears (Coro and Kössl, 1998; Kössl and Boyan, 1998), indicating a dynamic component to their response. These ears, like the Drosophila Johnston's organ, have chordotonal sensory cells. Together with axonemal defects in the $t i l B$ and $b t v$ mutants, they support the idea that active force generation by chordotonal cilia is involved in normal acoustic transduction.

\section{REFERENCES}

Adams CM, Anderson MG, Motto DG, Price MP, Johnson WA, Welsh MJ (1998) Ripped pocket and Pickpocket, novel Drosophila DEG/ENaC subunits expressed in early development and in mechanosensory neurons. J Cell Biol 140:143-152.

Bennet-Clark HC (1971) Acoustics of insect song. Nature 234:255-259.

Bennet-Clark HC, Ewing AW (1969) Pulse interval as a critical parameter in the courtship song of Drosophila melanogaster. Anim Behav 17:755-759.

Bodmer R, Jan YN (1987) Morphological differentiation of the embryonic peripheral neurons in Drosophila. Roux's Arch Dev Biol 196:69-77.

Burkhardt D, Gewecke M (1965) Mechanoreception in Arthropoda: the chain from stimulus to behavioral pattern. Cold Spring Harb Symp Quant Biol 30:601-614.

Carlson SD, Hilgers SL, Juang J-L (1997) First developmental signs of the scolopale (glial) cell and neuron comprising the chordotonal organ in the Drosophila embryo. Glia 19:269-274.

Colley N, Baker E, Stamnes M, Zuker C (1991) The cyclophilin homolog ninaA is required in the secretory pathway. Cell 67:255-263.

Coro F, Kössl M (1998) Distortion product otoacoustic emissions from the tympanic organ in two noctuid moths. J Comp Physiol [A] 183:525-531. Dallos P (1992) The active cochlea. J Neurosci 12:4575-4585.

Darboux I, Lingueglia E, Pauron D, Barbry P, Lazdunski M (1998) A new member of the amiloride-sensitive sodium channel family in Drosophila melanogaster peripheral nervous system. Biochem Biophys Res Commun 246:210-216.

Driscoll M, Chalfie M (1991) The mec-4 gene is a member of a family of
Caenorhabditis genes that can mutate to induce neuronal degeneration. Nature 349:588-593.

Eberl DF (1999) Feeling the vibes: chordotonal mechanisms in insect hearing. Curr Opin Neurobiol 9:389-393.

Eberl DF, Duyk GM, Perrimon N (1997) A genetic screen for mutations that disrupt an auditory response in Drosophila melanogaster. Proc Natl Acad Sci USA 94:14837-14842.

Ewing AW (1978) The antenna of Drosophila as a "love song" receptor. Physiol Entomol 3:33-36.

FlyBase (1999) The FlyBase database of the Drosophila genome projects and community literature. Available from http://flybase.bio.indiana.edu/. Nucleic Acids Res 27:85-88.

Gupta BP, Rodrigues V (1997) Atonal is a proneural gene for a subset of olfactory sense organs in Drosophila. Genes Cells 2:225-233.

Hallberg E (1984) Paracrystalline inclusions in the cilia of the sensory cells in Johnston's organ of Calliphora erythrocephala Meigen (Diptera: Calliphoridae). Int J Insect Morphol Embryol 13:77-79.

Huang M, Gu G, Ferguson EL, Chalfie M (1995) A stomatin-like protein necessary for mechanosensation in C. elegans. Nature 378:292-295.

Jarman AP, Grau Y, Jan LY, Jan YN (1993) atonal is a proneural gene that directs chordotonal organ formation in the Drosophila peripheral nervous system. Cell 73:1307-1321.

Jarman AP, Grell EH, Ackerman L, Jan LY, Jan YN (1994) atonal is the proneural gene for Drosophila photoreceptors. Nature 369:398-400.

Jarman AP, Sun Y, Jan LY, Jan YN (1995) Role of the proneural gene, atonal, in formation of Drosophila chordotonal organs and photoreceptors. Development 121:2019-2030.

Kaplan J, Horvitz H (1993) A dual mechanosensory and chemosensory organ in Caenorhabditis elegans. Proc Natl Acad Sci USA 90:2227-2231.

Keil TA (1997) Functional morphology of insect mechanoreceptors. Microsc Res Tech 39:506-531.

Kernan M (1997) The molecular basis of the mechanical senses: one mechanism or many? J NIH Res 9:32-36.

Kernan M, Cowan D, Zuker C (1994) Genetic dissection of mechanosensory transduction: mechanoreception-defective mutations of Drosophila. Neuron 12:1195-1206.

Kössl M, Boyan G (1998) Acoustic distortion products from the ear of a grasshopper. J Acoust Soc Am 104:326-335.

Kyriacou CP, Hall JC (1986) Interspecific genetic control of courtship song production and reception in Drosophila. Science 232:494-497.

Manning A (1967a) Antennae and sexual receptivity in Drosophila melanogaster females. Science 158:136-137.

Manning A (1967b) The control of sexual receptivity in female Drosophila. Anim Behav 15:239-250.

Merritt J (1997) Transformation of external sensilla to chordotonal sensilla in the cut mutant of Drosophila assessed by single-cell marking in the embryo and larva. Microsc Res Tech 39:492-505.

Moran D, Varela F, Rowley J (1977) Evidence for active role of cilia in sensory transduction. Proc Natl Acad Sci USA 74:793-797.

Moulins M (1976) Ultrastructure of chordotonal organs. In: Structure and function of proprioceptors in the invertebrates (Mill PJ, ed), pp 387-426. London: Chapman and Hall.

Pak W, Grossfield J, White N (1969) Nonphototactic mutants in a study of vision of Drosophila. Nature 222:351-354.

Perkins LA, Hedgecock EM, Thomson JN, Culotti JG (1986) Mutant sensory cilia in the nematode Caenorhabditis elegans. Dev Biol 117:456-487.

Shanbhag SR, Singh K, Singh RN (1992) Ultrastructure of the femoral chordotonal organs and their novel synaptic organization in the legs of Drosophila melanogaster Meigen (Diptera: Drosophilidae). Int J Insect Morphol Embryol 21:311-322.

Tavernarakis N, Driscoll M (1997) Molecular modeling of mechanotransduction in the nematode Caenorhabditis elegans. Annu Rev Physiol 59:659-689.

Thurm U (1964) Mechanoreceptors in the cuticle of the honey bee: fine structure and stimulus mechanism. Science 145:1063-1065.

Tischner H (1953) Über den Gehörsinn von Stechmücken. Acustica 3:335-343.

Tokuyasu K, Peacock W, Hardy R (1972) Dynamics of spermiogenesis in Drosophila melanogaster. I. Individualization process. Z Zellforsch 124:479-506.

Uga S, Kuwabara M (1965) On the fine structure of the chordotonal sensillum in antenna of Drosophila melanogaster. J Electron Microsc (Tokyo) 14:173-181.

von Schilcher F (1976) The function of pulse song and sine song in the courtship of Drosophila melanogaster. Anim Behav 24:622-625.

Walker R, Willingham A, Zuker C (2000) A Drosophila mechanosensory transduction channel. Science 287:2229-2234.

Wolbarsht ML (1960) Electrical characteristics of insect mechanoreceptors. J Gen Physiol 44:105-122.

Wolbarsht ML, Dethier VG (1958) Electrical activity in the chemoreceptors of the blowfly. I. Responses to chemical and mechanical stimulation. J Gen Physiol 42:393-412. 